\title{
Transcription Initiation Factor TFIID Subunit 12 Isoform TAFII15
}

National Cancer Institute

\section{Source}

National Cancer Institute. Transcription Initiation Factor T FIID Subunit 12 Isoform

TAFII15. NCI Thesaurus. Code C30061.

Transcription initiation factor TFIID subunit 12 TAFII15 (131 aa, $15 \mathrm{kDa}$ ) is encoded by the human TAF12 gene. This protein plays a role in the regulation of both histone acetylation and gene transcription. 